\title{
Tidal dynamics of transiting exoplanets
}

\author{
Daniel C. Fabrycky ${ }^{1}$ \\ ${ }^{1} \mathrm{UCO} /$ Lick University of California Santa Cruz, CA 95064 \\ email: daniel.fabrycky@gmail.com
}

\begin{abstract}
Transits give us the mass, radius, and orbital properties of the planet, all of which inform dynamical theories. Two properties of the hot Jupiters suggest they had a dramatic origin via tidal damping from high eccentricity. First, the tidally circularized planets (in the 1-4 day pile-up) lie along a relation or boundary in the mass-period plane. This observation may implicate a tidal damping process regulated by planetary radius inflation and Roche lobe overflow, early in the planets' lives. Second, the host stars of many planets have spins misaligned from the planets' orbits. This observation was not expected a priori from the conventional disk migration theory, and it was a boon for the alternative theories of planet-planet scattering and Kozai cycles, accompanied by tidal friction, which predicted it. Now we are faced with a curious observation that the misalignment angle depends on the stellar temperature. It may mean that the tide raised on the stars realigns them, the final result being the tidal consumption of hot Jupiters.
\end{abstract}

Keywords. planetary systems: formation, eclipses

\section{Introduction}

Two of the central mysteries of extrasolar gas giants is that they have small periods and large eccentricities, relative to the giants of the solar system (see Figure 1). A host of physical processes may account for these differences, but the most ubiquitous and robust mechanisms seem to be migration in a gas disk to shorten orbital periods (Goldreich \& Tremaine 1980; Ward 1997; Lubow \& Ida 2010) and chaotic interactions among planets to increase orbital eccentricities (Jurić \& Tremaine 2008; Chatterjee et al. 2008; Ford \& Rasio 2008). At first glance then, it may seem that the orbital evolution of hot Jupiters - a class of planets with typical periods 1-4 days and nearly circular orbits, and usually no known companion planets (Wright et al. 2009) - is dominated by migration in the gas disk with not by dynamical interactions. However, orbits with periapses as close to the star as these would tend to circularize via tidal dissipation. Therefore, it is possible that hot Jupiters actually arise from dramatic eccentricity excitation without much migration in the disk, after which the orbits circularize tidally (Rasio \& Ford 1996; Wu \& Murray 2003; Matsumura et al. 2010). These two vastly different evolutionary paths are illustrated on Figure 1. The latter mechanism has a sharp distance dependence - if it happens at all, it must result in a characteristic orbital period, i.e. it could lead to the observed 3-day pile-up (Wu et al. 2007), as illustrated by Figure 2 .

Transiting planets offer some new possibilities for resolving the origin of hot Jupiters because the orbital and physical properties can be precisely measured. Furthermore, unlike in the case of radial-velocity discoveries which yields $m \sin i$ of the planet, the true mass of transiting planets can be measured. These measurements have revealed an intriguing trend among the hot Jupiters: planets with periods $\sim 1$ day are more massive than their $\sim 3$ day brethren. Section 2 is devoted to this statistical result and a potential explanation. Another property of the orbit, its alignment relative to the star's 
spin, is only measurable via transits, using the Rossiter-McLaughlin effect (Winn, these proceedings). Thus transits offer a new property for discerning planetary migration, and this is discussed in section 3 .

\section{Circular hot Jupiters follow a mass-period relation}

After only a few transiting planets were discovered, Mazeh et al. (2005) boldly identified a relationship between the planetary masses and orbital periods: for the hot Jupiters, they were inversely correlated. As more transiting planets were discovered, it came to be characterized more as a lower limit to mass as a function of orbital period, rather than a relation. That is, massive exoplanets were found to transit and were far off the relation. These same planets are typically more eccentric, being less prone to tidal dissipation in the planet, which causes circularization. After separating the planets into those that are robustly eccentric and those that are consistent with circular, Pont et al. (2011) (see Husnoo et al. these proceedings) found that the circular orbits still follow an anti-correlation in mass versus period.

This observation suggests that being drawn on to the mass-period relation might be considered a fundamental aspect of hot Jupiters, that some property of their origin establishes circular orbits on the mass-period relation. Tidal dissipation in the planet can indeed bring planets to circular orbits, and the period does shrink as the orbit circularizes, but the end point of standard tidal dissipation does not depend on the planet's mass. However, it has been recognized (e.g. Gu et al. 2003; Gaudi et al. 2005) that hot Jupiters have orbital energies greatly exceeding their binding energy, and that this can lead to planetary inflation, mass loss, and even tidal disruption. In particular, if a planet dissipates from a very high eccentricity orbit (much larger semi-major axis $a$ ), then the tidal energy input is:

$$
E_{\mathrm{t}}=-E_{\mathrm{orb}}=G M_{\star} M_{p} /(2 a),
$$

whereas the binding energy of the planet is:

$$
E_{\mathrm{b}}=k G M_{p}^{2} / R_{p}
$$

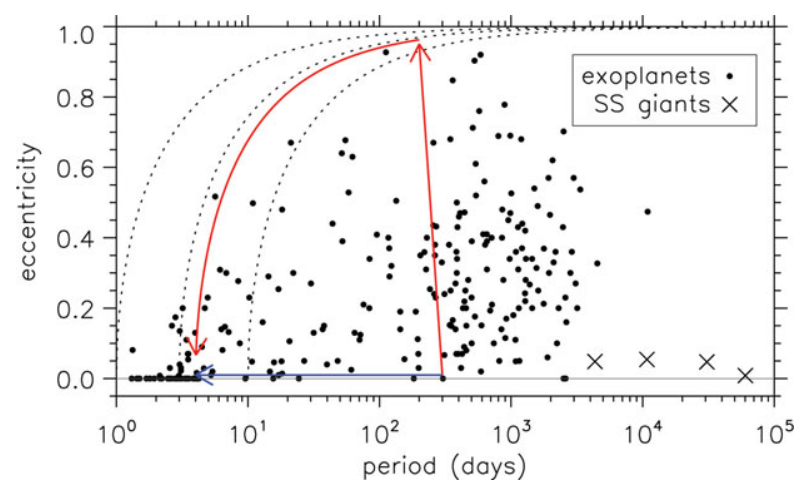

Figure 1. Periods and eccentricities of exoplanets, which differ markedly from the giants of the solar system. Broadly speaking, two distinct pathways for forming hot Jupiters are shown by the arrows. Lines of constant angular momentum (dotted) show the paths of exoplanets that are evolving by tides in the planet alone, with negligible angular momentum transferred to the planet's spin. The large population of tidally circularized hot Jupiters is readily apparent. 
where at an age of $10^{6}-10^{7} \mathrm{Myr}, k \approx 1$ and $R_{p}=1.3-1.6 R_{\mathrm{Jup}}$ (Burrows et al. 2000; Marley et al. 2007). Thus, overflow is energetically possible $\left(E_{\mathrm{t}} \gtrsim E_{\mathrm{b}}\right)$ if

$$
M_{p} \lesssim 19.2 M_{\text {Jup }}\left(M_{\star} / M_{\odot}\right)^{2 / 3}(P / \text { day })^{-2 / 3} \text {. }
$$

Curiously, this mass-period limit lies parallel (although much higher in mass than) the mass-period relation of Mazeh et al. (2005).

Energetically, then, all the circularized hot Jupiters might have gone through an epoch of tidal inflation and/or overflow. If the initial perhaps separation is at the tidal radius or below $\left(\lesssim 2.4\left(m_{\star} / m_{p}\right)^{1 / 3} R_{p}\right)$ then the planet will be ripped apart on one or a few orbital periods (Faber et al. 2005). If it starts slightly further out, the radius inflation still meets the Roche surface with unstable results (Guillochon et al. 2011).

However, if the initial periapse is distant enough, this process could be rather gentle, tearing off only a very small fraction of the mass per orbital period. The lionshare of the mass will be lost through the (instantaneous) inner Lagrangian point, towards the star. The gas would form an accretion disk around the star, transferring the mass to the star and transporting angular momentum back out, ultimately back to the planet. Therefore, even as the planet loses mass, it will nearly conserve its angular momentum. The result is likely capable of drawing the planet to the mass-period relation. In particular, lower mass gas giants are more susceptible to tidal dissipation and radius inflation, and they will thus lose a larger fraction of their mass, and gain more specific angular momentum through mass transfer.

\section{Spin-orbit misalignment}

Most mechanisms that can set up such extreme eccentricities, such that the hypothesis of the previous section is applicable, are also likely to excite inclinations. Let us first suppose that a planetary system begins aligned with its host star's spin, as both orientations are set by the protoplanetary accretion disk (but see below). Disk migration would presumably maintain this alignment. In contrast, the mechanisms of Kozai cycles

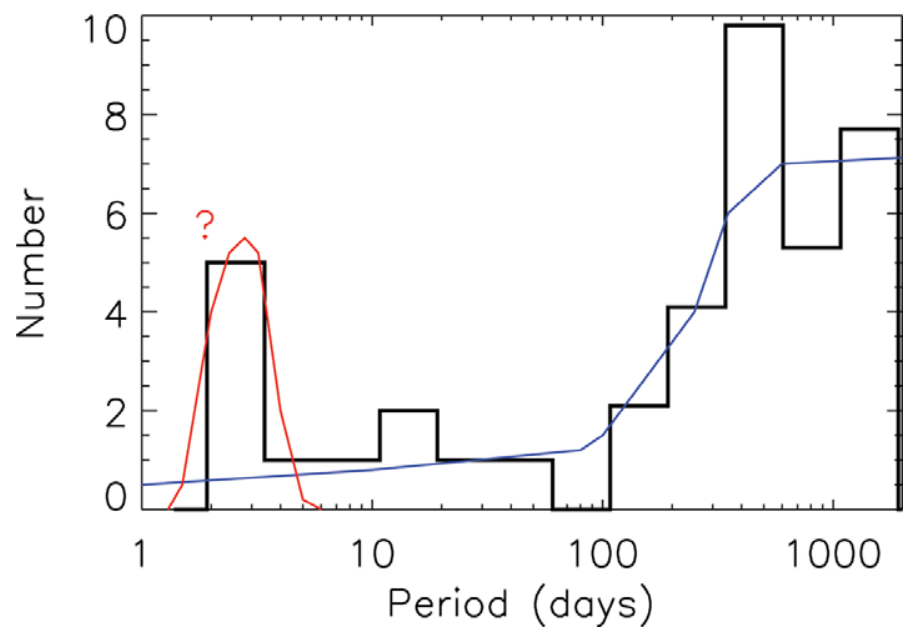

Figure 2. Empirical period distribution of gas-giant $\left(m \sin i>0.3 \mathrm{M}_{\mathrm{Jup}}\right.$ ) exoplanets (Cumming et al. 2008). There is a clear probability enhancement at short orbital periods: the hot Jupiters are piled up at 3 days. Perhaps disk migration creates a smooth component that populates from $\sim 1$ AU to the stellar surface with declining efficiency, whereas tidal dissipation from high eccentricity creates the pile-up. 
due to a binary companion (Fabrycky \& Tremaine 2007; Wu et al. 2007) and planetplanet scattering (Nagasawa et al. 2008) have been shown to yield wide distributions of spin-orbit misalignment. Dramatically, these mechanisms predict that some hot Jupiters should be on retrograde orbits, that is, the stellar obliquity should be $>90$ degrees.

Therefore the second probe of migration specific to transit measurements is the angle between planetary orbits and stellar spins, observable during transits through the spectroscopic anomaly known as the Rossiter-McLaughlin effect (Queloz et al. 2000; Gaudi \& Winn 2007; Fabrycky \& Winn 2009, Winn in this volume). Over the past two years, a large statistical sample has been built up by very busy observers (e.g. Triaud et al. 2010; Winn et al. 2010). Both a large population of aligned systems, as well as large prograde inclinations and retrograde inclinations, have all been observed. In fact, the fraction of highly inclined and retrograde systems are in rough agreement with the fraction predicted by the mechanisms forming hot Jupiters via high eccentricities (Morton \& Johnson 2011). However, one difficulty with this hypothesis is that it does not leave enough very nearly aligned systems (Fabrycky \& Winn 2009) - this "core" of well-aligned systems is a robust feature, despite the deluge of misaligned systems.

Another curious fact is that the hot stars (defined as $T_{\text {eff }}>6250 \mathrm{~K}$ ) are much more often to have misaligned planets (Winn et al. 2010). In fact, just these stars are completely consistent with strong scattering prediction (Morton \& Johnson 2011). Perhaps all hot Jupiters arrive via high eccentricity and inclination, and the cool stars are somehow able to damp those inclinations to quite small values. In particular, tidal dissipation in the cool stars, which have more dynamically significant convective envelopes, seems a likely damping mechanism. Moreover, among the cool stars, there are some misalignments, and these are the systems for which the planets raise the weakest tide on their stars. See (Winn et al. 2010) and Winn in this volume.

Despite all these empirical encouragements, the theory of how tides can damp inclinations seems problematic. When one tries to construct a tidal model of tides raised on stars, one immediately finds that the large inertia of stars means that a typical hot Jupiter cannot torque its star into spin-orbit synchrony and survive the process - it will surrender all its orbital angular momentum as it spins up the star (Rasio et al. 1996; Levrard et al. 2009; Jackson et al. 2009; Taylor 2010). In fact, the inclination damping timescale and planetary consumption timescale rely on similar physics, so one expects that moderate-mass exoplanets would only reorient their stars as they plunge in (Barker \& Ogilvie 2009).

What is needed is a mechanism that allows the star to reorient without spinning up its bulk, otherwise it would rob the planet of too much angular momentum. Thus Winn et al. (2010) proposed the following resolution: the spin rate of the interior radiative zone decouples from the outer convective zone. The outer parts of the star, which are most strongly affected by tides from the planet, are free to respond to it. They are also free to be torqued down by the magnetic wind, assuring that the surface rotation does not violate the observation that hot-Jupiter hosts are not particularly fast surface rotators. This concept of differential rotation within solar-type stars is not new; it is a natural consequence of magnetic wind torque preferentially slowing the outer parts of the star (Pinsonneault et al. 1989), and it has received some empirical support through observations of rotation periods in young stellar clusters (Irwin \& Bouvier 2009). Several mechanisms act to restore near-solid-body rotation: hydrodynamic instabilities (Goldreich \& Schubert 1967), g-modes (Zahn et al. 1997), and magnetic torques. One or all of which have apparently coupled the radiative interior and the convective zone of the Sun (Howe 2009). On that coupling timescale, the hot Jupiter would interact with the whole star and tidally spiral in. So if this picture is correct, it implies that most 
of the hot Jupiters with spin-orbit alignment with cool stars are in a transient state, and they will meet a spectacular end through tidal consumption.

Finally, we must address whether spin-orbit misalignments are proof that some hot Jupiters arrive via high eccentricities. On the contrary: after the discovery of several retrograde planets, the assumptions of spin-orbit alignment resulting from disk migration were questioned by several authors. Bate et al. (2010) suggested that the host star simply does not need to be aligned with the disk that formed the planets, as its spin orientation represents the whole build-up of mass, accreted from many directions within the birth cloud, whereas the planets are probably built from only the last material that was accreted into a disk. Lai et al. (2011) and Foucart \& Lai (2011), on the other hand, described a model of protostellar accretion in which the magnetic interaction between the star and the disk can amplify the inclination difference between them. If either of these mechanisms, or additional mechanisms, can explain the correlation between spin-orbit misalignment and stellar temperature, then disk migration may regain its full appeal as the mechanism that produces most hot Jupiters.

\section{Conclusions}

Transiting planets have offered interesting new windows into the origin of hot Jupiters, the enigmatic set of gas-giant planets parked at few-day periods from their stars. Continued increases of the samples of hot Jupiters, and more investigation into trends and correlations of spin-orbit alignment, are mandatory before these issues will be completely resolved. It is quite likely that hot Jupiters come from some combination of the evolutionary paths shown in Figure 1, and the relative contribution is what needs to be determined. But some universal properties of hot Jupiters, e.g. the mass-period correlation and misalignments preferentially around hot stars, may indicate that future theoretical work may be able to interpret these observational trends within a comprehensive and singular framework.

\section{References}

Barker, A. J. \& Ogilvie, G. I. 2009, MNRAS, 395, 2268

Bate, M. R., Lodato, G., \& Pringle, J. E. 2010, MNRAS, 401, 1505

Burrows, A., Guillot, T., Hubbard, W. B., Marley, M. S., Saumon, D., Lunine, J. I., \& Sudarsky, D. 2000, ApJL, 534, L97

Chatterjee, S., Ford, E. B., Matsumura, S., \& Rasio, F. A. 2008, ApJ, 686, 580

Cumming, A., Butler, R. P., Marcy, G. W., Vogt, S. S., Wright, J. T., \& Fischer, D. A. 2008, $P A S P, 120,531$

Faber, J. A., Rasio, F. A., \& Willems, B. 2005, Icarus, 175, 248

Fabrycky, D. \& Tremaine, S. 2007, ApJ, 669, 1298

Fabrycky, D. C. \& Winn, J. N. 2009, ApJ, 696, 1230

Ford, E. B. \& Rasio, F. A. 2008, ApJ, 686, 621

Foucart, F. \& Lai, D. 2011, MNRAS, 234

Gaudi, B. S., Seager, S., \& Mallen-Ornelas, G. 2005, ApJ, 623, 472

Goldreich, P. \& Schubert, G. 1967, ApJ, 150, 571

Gaudi, B. S. \& Winn, J. N. 2007, ApJ, 655, 550

Goldreich, P. \& Tremaine, S. 1980, ApJ, 241, 425

Gu, P.-G., Lin, D. N. C., \& Bodenheimer, P. H. 2003, ApJ, 588, 509

Guillochon, J., Ramirez-Ruiz, E., \& Lin, D. N. C. 2011, ApJ, 732, id.74

Howe, R. 2009, Living Reviews in Solar Physics, 6, 1

Irwin, J. \& Bouvier, J. 2009, in IAU Symposium, Vol. 258, ed. E. E. Mamajek, D. R. Soderblom, \& R. F. G. Wyse, 363 
Jackson, B., Barnes, R., \& Greenberg, R. 2009, ApJ, 698, 1357

Jurić, M. \& Tremaine, S. 2008, ApJ, 686, 603

Lai, D., Foucart, F., \& Lin, D. N. C. 2011, MNRAS, 231

Lubow, S. H. \& Ida, S. 2010, Planet Migration, in EXOPLANETS, pg. 347-371, ed. S. Seager, University of Arizona Press

Levrard, B., Winisdoerffer, C., \& Chabrier, G. 2009, ApJL, 692, L9

Marley, M. S., Fortney, J. J., Hubickyj, O., Bodenheimer, P., \& Lissauer, J. J. 2007, ApJ, 655, 541

Matsumura, S., Peale, S. J., \& Rasio, F. A. 2010, ApJ, 725, 1995

Mazeh, T., Zucker, S., \& Pont, F. 2005, MNRAS, 356, 955

Morton, T. D. \& Johnson, J. A. 2011, ApJ, 729, 138

Nagasawa, M., Ida, S., \& Bessho, T. 2008, ApJ, 678, 498

Pinsonneault, M. H., Kawaler, S. D., Sofia, S., \& Demarque, P. 1989, ApJ, 338, 424

Pont, F., Husnoo, N., Mazeh, T., \& Fabrycky, D. 2011, MNRAS, 414, 1278

Queloz, D., Eggenberger, A., Mayor, M., Perrier, C., Beuzit, J. L., Naef, D., Sivan, J. P., \& Udry, S. 2000, A\& $A, 359$, L13

Rasio, F. A., Tout, C. A., Lubow, S. H., \& Livio, M. 1996, ApJ, 470, 1187

Rasio, F. A. \& Ford, E. B. 1996, Science, 274, 954

Taylor, S. F. 2010, preprint arXiv:1009.4221

Triaud, A. H. M. J., Collier Cameron, A., Queloz, D., Anderson, D. R., Gillon, M., Hebb, L., Hellier, C., Loeillet, B., Maxted, P. F. L., Mayor, M., Pepe, F., Pollacco, D., Ségransan, D., Smalley, B., Udry, S., West, R. G., \& Wheatley, P. J. 2010, A\&\&A, 524, A25+

Ward, W. R. 1997, ApJL, 482, L211+

Winn, J. N., Fabrycky, D., Albrecht, S., \& Johnson, J. A. 2010, ApJL, 718, L145

Wright, J. T., Upadhyay, S., Marcy, G. W., Fischer, D. A., Ford, E. B., \& Johnson, J. A. 2009, ApJ, 693, 1084

Wu, Y. \& Murray, N. 2003, ApJ, 589, 605

Wu, Y., Murray, N. W., \& Ramsahai, J. M. 2007, ApJ, 670, 820

Zahn, J.-P., Talon, S., \& Matias, J. 1997, A\&A, 322, 320 